\title{
BMJ Open Trends in the prescription of systemic anticancer therapy and mortality among patients with advanced non-small cell lung cancer: a real-world retrospective observational cohort study from the I-O optimise initiative
}

To cite: Snee M, Cheeseman S, Thompson M, et al. Trends in the prescription of systemic anticancer therapy and mortality among patients with advanced non-small cell lung cancer: a real-world retrospective observational cohort study from the I-0 optimise initiative. BMJ Open 2021;11:e043442. doi:10.1136/ bmjopen-2020-043442

- Prepublication history and additional supplemental material for this paper are available online. To view these files, please visit the journal online (http://dx.doi.org/10.1136/ bmjopen-2020-043442).

Received 11 August 2020 Revised 03 February 2021 Accepted 17 March 2021

Check for updates

(c) Author(s) (or their employer(s)) 2021. Re-use permitted under CC BY-NC. No commercial re-use. See rights and permissions. Published by BMJ.

For numbered affiliations see end of article.

Correspondence to

Dr Michael Snee;

m.snee@nhs.net

\section{ABSTRACT}

Objectives To assess how a decade of developments in systematic anticancer therapy (SACT) for advanced nonsmall cell lung cancer (NSCLC) affected overall survival (OS) in a large UK University Hospital.

Design Real-world retrospective observational cohort study using existing data recorded in electronic medical records.

Setting A large National Health Service (NHS) university teaching hospital serving 800000 people living in a diverse metropolitan area of the UK.

Participants 2119 adults diagnosed with advanced NSCLC (tumour, node, metastasis stage IIIB or IV) between 2007 and 2017 at Leeds Teaching Hospitals NHS Trust. Main outcomes and measures OS following diagnosis and the analysis of factors associated with receiving SACT. Results Median OS for all participants was 2.9 months, increasing for the SACT-treated subcohort from 8.4 months (2007-2012) to 9.1 months (2013-2017) $(p=0.02) ; 1$-year OS increased from $33 \%$ to $39 \%$ over the same period for the SACT-treated group. Median OS for the untreated subcohort was 1.6 months in both time periods. Overall, $30.6 \%$ (648/2119) patients received SACT; treatment rates increased from $28.6 \%(338 / 1181)$ in 2007-2012 to $33.0 \%$ (310/938) in 2013-2017 ( $p=0.03)$. Age and performance status were independent predictors for SACT treatment; advanced age and higher performance status were associated with lower SACT treatment rates.

Conclusion Although developments in SACT during 2007-2017 correspond to some changes in survival for treated patients with advanced NSCLC, treatment rates remain low and the prognosis for all patients remains poor.

\section{INTRODUCTION}

Survival for UK patients with lung cancer has historically been poor compared with similar Western nations. ${ }^{12}$ In 2017, approximately $65 \%$ of patients in England and Wales with pathologically confirmed advanced stage
Strengths and limitations of this study

- This retrospective observational cohort study analysed a long-term real-world dataset of patients with advanced non-small cell lung cancer from a large UK regional hospital.

- This hospital database contained detailed information on the clinical characteristics of patients including their performance status and on the systematic anticancer therapy (SACT) regimens received.

- Data are reported from a single site; however, the cohort analysed was large and drawn from a reasonably representative population of UK patients.

- Factors associated with the likelihood of SACT treatment are presented; however, a more detailed clinical case review would be required to confirm why these factors impact decision to treat.

non-small cell lung cancer (NSCLC) and good Eastern Cooperative Oncology Group performance status (PS) received systematic anticancer therapy (SACT). ${ }^{3}$

For decades, platinum-based chemotherapy has been the mainstay of SACT. Chemotherapy provides only a modest survival benefit versus palliative care and has notable toxicity. In the Big Lung Trial, median overall survival (OS) was extended by about 9 weeks with cisplatin-based chemotherapy compared with supportive care $(p=0.0006)$ in a patient population with primarily $(95 \%)$ stage III-IV NSCLC. Toxicity levels were similar to other cisplatin-based regimens (31\% grade 3-4 toxicity). ${ }^{4}$ Recent treatment advances have led to the introduction of tyrosine kinase inhibitors (TKIs) and immune checkpoint inhibitors, targeting the programmed death 
receptor 1 /programmed death ligand 1 (PD-1/PD-L1) pathway. ${ }^{5-7}$ In clinical trials, these SACT classes have demonstrated superiority to chemotherapy in response rates, tolerability and OS for selected patients. ${ }^{6-8}$

In the UK, the TKI gefitinib has been available (recommended by National Institute for Health and Care Excellence (NICE)) since July 2010 for first-line (1L) and July 2009 for second-line (2L) therapy in patients who have an epidermal growth factor receptor (EGFR) mutation. Several new TKIs became available during the study period. Erlotinib, which also targets mutant EGFR, was first recommended by NICE in June 2012 for 1L treatment in patients with the EGFR mutation and November 2008 for $2 \mathrm{~L}$ treatment in all patients with NSCLC. However, the indication for 2L was updated in December 2015 to only include patients with an EGFR mutation (or unknown status). Another EGFR inhibitor, afatinib, has been available since April 2014 for $1 \mathrm{~L}$ and $2 \mathrm{~L}$ treatment in patients with an EGFR mutation. Osimertinib is indicated for $2 \mathrm{~L}$ therapy in patients who have progressed after receiving an EGFR-targeting TKI. Crizotinib and ceritinib have been recommended in the UK for $1 \mathrm{~L}$ treatment in patients with an ALK mutation since September 2016 and January 2018, respectively, and for 2L treatment from December 2016 and June 2016, respectively. Nintedanib in combination with docetaxel has been available in the UK since July 2015 for treatment of all patients with NSCLC.

The first two anti-PD-1 immune checkpoint inhibitors, pembrolizumab and nivolumab, have been available since 2017 as 2L therapies in patients with NSCLC. They are indicated by NICE for patients with NSCLC with PD-L1 expression $\geq 1 \%$ without ALK/EGFR aberrations. Nivolumab is also indicated in squamous NSCLC without PD-L1 expression. The first immune checkpoint inhibitor (pembrolizumab) for 1L therapy was available in July 2018, after the end of our study period.

In this study, we assessed the evolution in advanced NSCLC management and OS over the 2007-2017 period, prior to the launch of immune checkpoint inhibitors, at a large teaching hospital in the UK. The study is part of the I-O Optimise programme, a multinational collaboration aimed at developing a research framework to provide insights into the evolving real-world management and outcomes of patients with thoracic malignancies through partnership with existing real-world data sources across Europe and Canada. ${ }^{9}$

\section{METHODS}

The study included adult patients (aged $\geq 18$ years) with an incident diagnosis of tumour, node, metastasis stage IIIB or IV NSCLC between January 2007 and August 2017 at Leeds Teaching Hospital NHS Trust, a regional cancer centre serving approximately 800000 people. Incident diagnosis of NSCLC was determined according to International Statistical Classification of Diseases and Related Health Problems, 10th revision code for malignant neoplasm of the trachea (C33) or malignant neoplasm of bronchus and lung (C34). We extracted data from Leeds Teaching Hospitals NHS Trust electronic medical records (EMRs), which included data on SACT, and pathology and radiology reports. All included patients were diagnosed by the lung multidisciplinary team: those clinically identified solely by history, examination and CT and those confirmed by pathology (International Classification of Diseases for Oncology, third edition (ICD-O-3) code for NSCLC histology). Data were clinically curated by checking for inconsistencies where they typically arise (such as diagnosis and treatment dates) and any noted issues were resolved. REAL-Oncology, a research partnership between Leeds Cancer Centre, the University of Leeds and IQVIA, was responsible for analysing the data using SAS V.9.4 (SAS Institute).

Patient demographics, PS and tumour pathology (ICDO-3 coding) data were extracted on/at the nearest date of NSCLC diagnosis (index date). Patients receiving SACT at any point following diagnosis were recorded as SACT treated. End of follow-up was date of death, end of study or end of available data from data source (due to loss to follow-up or known exit from data source). Date of death was confirmed by reconciliation of EMRs with Office for National Statistics death certifications. Minimum follow-up was 7 months. We split the study cohort into two consecutive time periods by diagnosis date (January 2007-December 2012 and January 2013-August 2017).

We calculated summary and descriptive statistics for categorical and continuous variables, and estimated OS from index date to date of death using Kaplan-Meier and log-rank tests. Patients without any record of death during the study were censored at the end of the study period (31 March 2018) or at the date of last available data (due to loss to follow-up or known exit from data source), whichever came first. We used Pearson's $\chi^{2}$ test of independence to identify unadjusted associations of categorical variables with SACT treatment. Following testing assumptions, we constructed a logistic regression model to estimate the effect of various prognostic variables on the probability of receiving SACT treatment (within 6 months of diagnosis). We included age, sex, pathology and stage covariates in a base model and added other potential prognostic factors in a stepwise manner. Goodness of fit was indicated using Akaike's Information Criterion (AIC) and the final most desirable parsimonious model was that with the lowest AIC.

\section{RESULTS}

Among the 2119 patients diagnosed with incident stage IIIB-IV NSCLC, median age at diagnosis was 72 years (range: $18-101) ; 84.1 \%(\mathrm{n}=1782)$ of patients had stage IV disease (table 1 and online supplemental appendix table $\mathrm{S} 1)$. At diagnosis, $31.2 \%(\mathrm{n}=662)$ of patients had PS $0-1$, $19.5 \%(n=414)$ had PS 2 and $40.0 \%(n=848)$ had PS $>2$; 9.2\% ( $\mathrm{n}=195)$ had PS data missing. The 2007-2012 and 2013-2017 subcohorts included 1181 and 938 patients, 
Table 1 Patient characteristics at diagnosis, overall and by treatment received in patients with incident stage IIIB-IV NSCLC

\begin{tabular}{|c|c|c|c|}
\hline & $\begin{array}{l}\text { All } \\
(\mathrm{N}=2119) \\
n(\%)\end{array}$ & $\begin{array}{l}\text { Untreated } \\
(n=1471) \\
n(\%)\end{array}$ & $\begin{array}{l}\text { SACT treated } \\
(\mathrm{n}=648) \\
\mathrm{n}(\%)\end{array}$ \\
\hline \multicolumn{4}{|l|}{ Age at diagnosis, years } \\
\hline$<65$ & $541(25.5)$ & $249(16.9)$ & $292(45.1)$ \\
\hline $65-74$ & $671(31.7)$ & $428(29.1)$ & $243(37.5)$ \\
\hline$\geq 75$ & $907(42.8)$ & $794(54.0)$ & $113(17.4)$ \\
\hline 2007-2012 & $1181(55.7)$ & $843(57.3)$ & $338(52.2)$ \\
\hline 2013-2017 & $938(44.3)$ & $628(42.7)$ & $310(47.8)$ \\
\hline \multicolumn{4}{|l|}{ Sex } \\
\hline Male & 1106 (52.2) & 755 (51.3) & $351(54.2)$ \\
\hline \multicolumn{4}{|l|}{ BMI } \\
\hline 18.5-24.9 (healthy weight) & $299(14.1)$ & $38(2.6)$ & $261(40.3)$ \\
\hline 25-29.9 (overweight) & $248(11.7)$ & $29(2.0)$ & $219(33.8)$ \\
\hline$\geq 30$ (obese) & $118(5.6)$ & $12(0.8)$ & $106(16.4)$ \\
\hline Missing/unknown & 1417 (66.9) & $1387(94.3)$ & $30(4.6)$ \\
\hline \multicolumn{4}{|l|}{ ECOG PS } \\
\hline 0 & $145(6.8)$ & $44(3.0)$ & $101(15.6)$ \\
\hline 1 & $517(24.4)$ & $231(15.7)$ & $286(44.1)$ \\
\hline 2 & $414(19.5)$ & $256(17.4)$ & $158(24.4)$ \\
\hline 3 & $564(26.6)$ & $518(35.2)$ & $46(7.1)$ \\
\hline Other & $49(2.3)$ & $20(1.3)$ & $29(4.5)$ \\
\hline NOS NSCLC & $315(14.9)$ & $201(13.7)$ & $114(17.6)$ \\
\hline Clinically diagnosed* & $693(32.7)$ & $668(45.4)$ & $25(3.8)$ \\
\hline \multicolumn{4}{|l|}{ Stage } \\
\hline IIIB & 337 (15.9) & 198 (13.5) & 139 (21.4) \\
\hline IV & 1782 (84.1) & 1273 (86.5) & 509 (78.6) \\
\hline
\end{tabular}

*No pathological confirmation. A total of 25 patients were recorded as receiving treatment following clinical diagnosis; however, further investigations confirmed that all these patients had pathologically confirmed NSCLC.

BMI, body mass index; ECOG, Eastern Cooperative Oncology Group; NOS, not otherwise specified; NSCLC, non-small cell lung cancer; NSQ, non-squamous; PS, performance status; ref, reference; SACT, systemic anticancer therapy; SQ, squamous.

respectively. Patient characteristics were similar between the two subcohorts.

Pathology of disease was non-squamous in $31.4 \%$ $(\mathrm{n}=666)$, squamous in $18.7 \%(\mathrm{n}=396)$ and not otherwise specified in $14.9 \%(\mathrm{n}=315)$ of patients; this was similar between the two subcohorts (online supplemental appendix table S1). Less common pathologies (adenoid cystic, sarcomatoid and miscellaneous) were identified in $2.3 \%(n=49)$ of patients, and $32.7 \%(n=693)$ of tumours were clinically identified but did not have pathological confirmation. Compared with patients with confirmed pathology, those without pathology were older (median 79 years) and less fit $(72.4 \%$ PS $>2$ vs $24.3 \%$ PS $>2$ for those with confirmed pathology; $\mathrm{p}<0.01)$.

Overall, $30.6 \% \quad(n=648)$ of patients were treated with SACT, and the unadjusted treatment rate was lower in the 2007-2012 subcohort $(28.6 \%, \mathrm{n}=338)$ versus the 20132017 subcohort $(33.0 \%, \mathrm{n}=310 ; \mathrm{p}=0.03)$. Specifically, the 


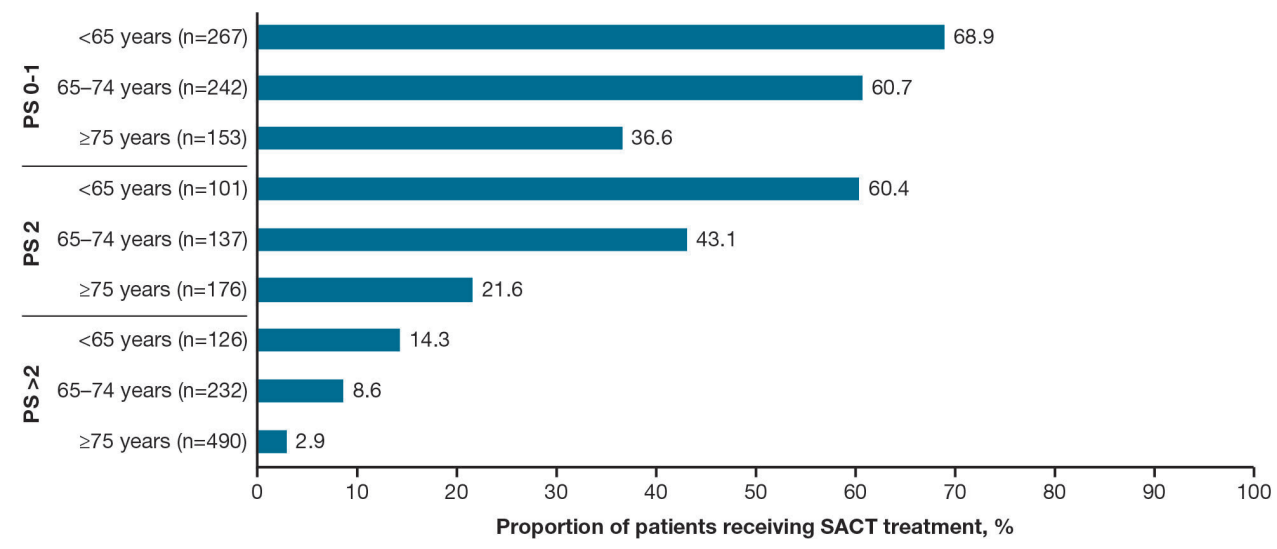

Figure 1 Proportion of patients with incident stage IIIB-IV receiving SACT treatment after NSCLC diagnosis, according to age and PS at diagnosis. NSCLC, non-small cell lung cancer; PS, performance status; SACT, systemic anticancer therapy.

treatment rate increased in the later period for patients aged $65-74$ years $(32.1 \%$ vs $41.3 \%$, respectively; $\mathrm{p}=0.01)$ and patients with PS $0-1$ (53.9\% vs $64.3 \%$, respectively; $\mathrm{p}<0.01)$; SACT treatment rates were similar between the two time periods subcohorts for other age and PS categories (data not shown).

SACT treatment rates decreased with increasing age and with higher PS (from $68.9 \%$ in patients aged $<65$ years with PS $0-1 \quad(n=184)$ to $2.9 \%(n=14)$ in patients aged $\geq 75$ years with PS $>2$; figure 1 ). Age group and PS were highly correlated $(p<0.01)$, but in the final logistic regression model, both factors appeared to be independent effect modifiers for the likelihood of SACT treatment. There was a decreasing trend in OR for SACT treatment with increasing age and with increasing PS (figure 2). The significant increase in SACT treatment in the 2013-2017 subcohort observed in the univariate analysis persisted in the multivariate model and there was also a lower OR of SACT treatment in patients with stage IV disease (compared with stage IIIB; figure 2). Treatment rates for patients with non-squamous and squamous pathology were very similar $(45.0 \% \quad(n=300)$ and $45.4 \%$ $(\mathrm{n}=180)$, respectively). Among the 693 clinically diagnosed patients, 25 patients were recorded as receiving SACT regimen following clinical diagnosis (3.6\%). Further investigation into these 25 patients revealed that all had, in fact, pathologically confirmed NSCLC, but this information was not recorded in their EMR leading to them being misclassified.

1L SACT was most commonly platinum-based combination chemotherapy $(84.7 \%, \mathrm{n}=549)$ or TKIs $(9.7 \%, \mathrm{n}=63$; table 2). Use of 1L TKIs increased between 2007-2012

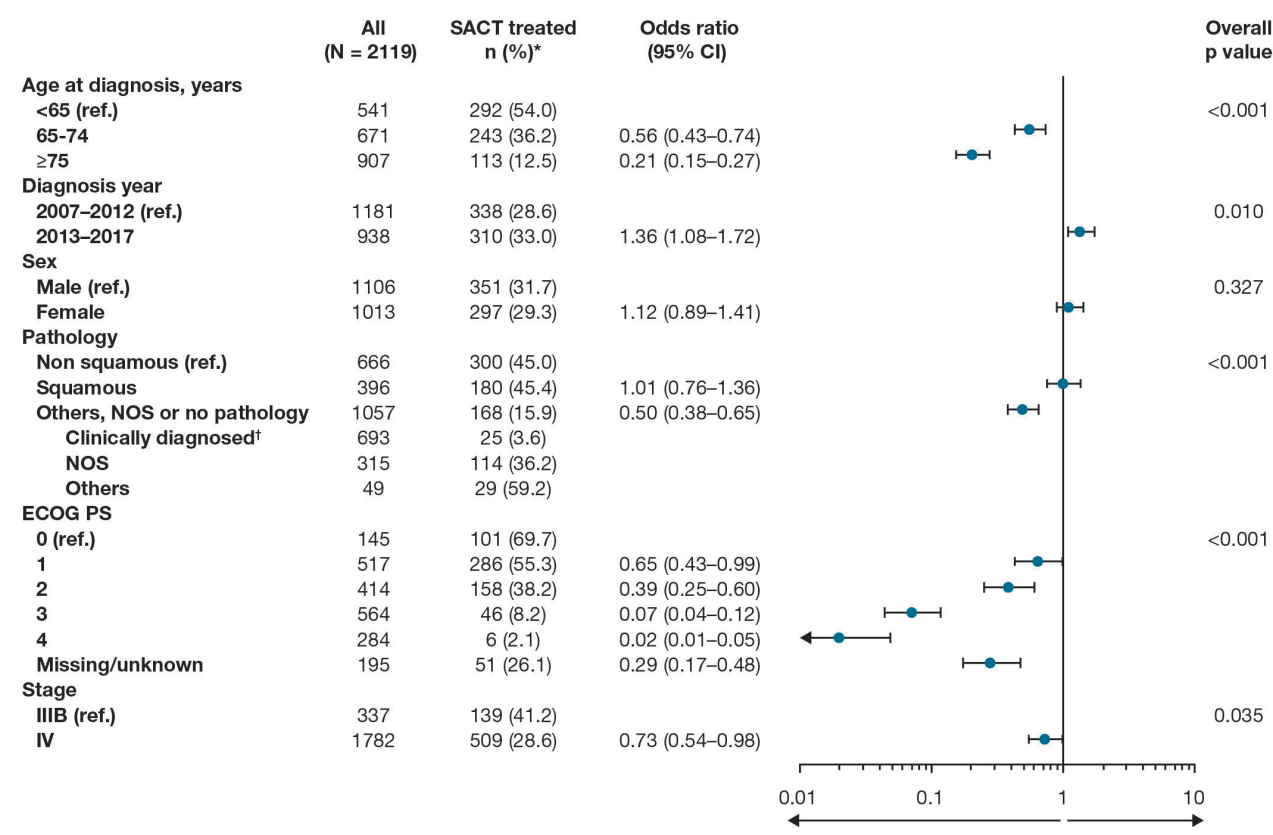

Figure 2 Logistic regression model for the odds of receiving SACT within 6 months of diagnosis among all incident stage IIIB-IV NSCLC. *Number and proportion of SACT-treated patients in each subpopulation. ${ }^{\dagger} A$ total of 25 patients were recorded as receiving treatment following clinical diagnosis, however, further investigations confirmed that all these patients had pathologically confirmed NSCLC. ECOG, Eastern Cooperative Oncology Group; NOS, not otherwise specified; NSCLC, nonsmall cell lung cancer; PS, performance status; ref., reference; SACT, systemic anticancer therapy. 
Table 2 SACT regimen received in $1 \mathrm{~L}$ and $2 \mathrm{~L}$ of therapy, overall and by time period in patients with incident stage IIIB-IV NSCLC at diagnosis

\begin{tabular}{|c|c|c|c|}
\hline & Overall & $2007-2012$ & 2013-2017 \\
\hline & $\mathbf{N}(\%)$ & n (\%) & n (\%) \\
\hline $1 \mathrm{~L}$ of therapy & 648 & 338 & 310 \\
\hline Platinum-based chemotherapy* & $549(84.7)$ & $292(86.4)$ & 257 (82.9) \\
\hline Carboplatin based & $456(70.4)$ & $258(76.3)$ & $198(63.9)$ \\
\hline Cisplatin based & $93(14.3)$ & $34(10.1)$ & $59(19.0)$ \\
\hline Pemetrexed containing treatments & $225(34.7)$ & $86(27.0)$ & $139(44.8)$ \\
\hline Non-platinum chemotherapy & $7(1.1)$ & $7(2.1)$ & 0 \\
\hline TKIs & $63(9.7)$ & $21(6.2)$ & $42(13.5)$ \\
\hline Gefitinib & $29(4.5)$ & $15(4.4)$ & $14(4.5)$ \\
\hline Erlotinib & $15(2.3)$ & $3(0.9)$ & $12(3.9)$ \\
\hline Afatinib & $13(2.0)$ & 0 & $13(4.2)$ \\
\hline Checkpoint inhibitors (anti-PD-L1) & $7(1.1)$ & 0 & $7(2.3)$ \\
\hline Pembrolizumab & $7(1.1)$ & 0 & $7(2.3)$ \\
\hline Clinical trial & $22(3.4)$ & $<20$ & $<5$ \\
\hline $2 \mathrm{~L}$ of therapy & 223 & 119 & 104 \\
\hline Platinum-based chemotherapy & $34(15.2)$ & $11(9.2)$ & $23(22.1)$ \\
\hline Non-platinum chemotherapy & $21(9.4)$ & $8(6.7)$ & $13(12.5)$ \\
\hline Docetaxel & $16(7.2)$ & $5(4.2)$ & $11(10.6)$ \\
\hline TKls & $141(63.2)$ & $99(83.2)$ & $42(40.4)$ \\
\hline Erlotinib & $113(50.7)$ & $96(80.7)$ & $17(16.3)$ \\
\hline Crizotinib & $7(3.1)$ & 0 & $7(6.7)$ \\
\hline Nintedanib + docetaxel & $10(4.5)$ & 0 & $10(9.6)$ \\
\hline Checkpoint inhibitors (anti-PD-L1) & $19(8.5)$ & 0 & 19 (18.3) \\
\hline Pembrolizumab & $10(4.5)$ & 0 & $10(9.6)$ \\
\hline Nivolumab & $7(3.1)$ & 0 & $7(6.7)$ \\
\hline Clinical trial & $6(2.7)$ & 0 & $6(5.8)$ \\
\hline
\end{tabular}

Patient numbers $<5$ have been masked; data are not shown for treatments with patient numbers $<5$ for all populations shown.

*Platinum-based chemotherapy included any regimen with a platinum agent as monotherapy or in combination, and was further defined as 'carboplatin based', 'cisplatin based' (including regimens where carboplatin and cisplatin were both used) and 'pemetrexed included' (any platinum-based regimen also including pemetrexed).

1L, first-line therapy; 2L, second-line therapy; NSCLC, non-small cell lung cancer; PD-L1, programmed death ligand 1; SACT, systematic anticancer therapy; TKIs, tyrosine kinase inhibitors.

$(6.2 \%, \mathrm{n}=21)$ and $2013-2017(13.5 \%, \mathrm{n}=42 ; \mathrm{p}<0.01)$ as platinum-based therapy use decreased (from $86.4 \%$, $\mathrm{n}=292$ in $2007-2012$ to $82.9 \%, \mathrm{n}=257$ in $2013-2017$; $\mathrm{p}=0.22$ ). Seven $(2.1 \%)$ patients in the $2007-2012$ subcohort received 1L non-platinum chemotherapy, and seven $(2.3 \%)$ patients in the 2013-2017 subcohort received $1 \mathrm{~L}$ immune checkpoint inhibitors. The remaining patients were treated with experimental clinical trial therapies.

Between the two treatment periods, a similar proportion of treated patients received 2L therapy $(35.2 \%$, $\mathrm{n}=119$ in $2007-2012$ compared with $33.5 \%, \mathrm{n}=104$ in 2013-2017; table 2). In patients receiving 2L therapy, we observed a decrease of 2L TKI use from $83.2 \%(n=99)$ in 2007-2012 to 40.4\% ( $\mathrm{n}=42)$ in 2013-2017. In 2013-2017, $18.3 \%(\mathrm{n}=19)$ of patients treated with 2L therapy received immunotherapy.
Median OS for the entire cohort was 2.9 months $(95 \%$ CI: 2.7 to 3.1 ) and 1 year OS was $17 \%$ (95\% CI: $15 \%$ to $19 \%$ ). Median OS was similar between 2007-2012 and 2013-2017 (2.8 vs 3.0 months, $\mathrm{p}=0.17$ ). Compared with patients not prescribed SACT, patients prescribed SACT had significantly longer median OS (8.7 vs 1.6 months, $\mathrm{p}<0.01)$ and 1 year OS (36\% vs $8 \%)$. OS for SACT-treated patients increased between 2007-2012 and 2013-2017, respectively (median OS 8.4 vs 9.1 months $(\mathrm{p}=0.02)$; 1-year OS $33 \%$ (95\% CI: $29 \%$ to $39 \%$ ) vs $39 \%$ (95\% CI: $34 \%$ to $45 \%$ ); 2- year OS $12 \%$ (95\% CI: $9 \%$ to $16 \%$ ) vs $22 \%$ (95\% CI: $17 \%$ to $27 \%$ ); figure 3 ).

\section{DISCUSSION}

In this study from a large university hospital, OS of patients presenting with advanced NSCLC was poor, with 


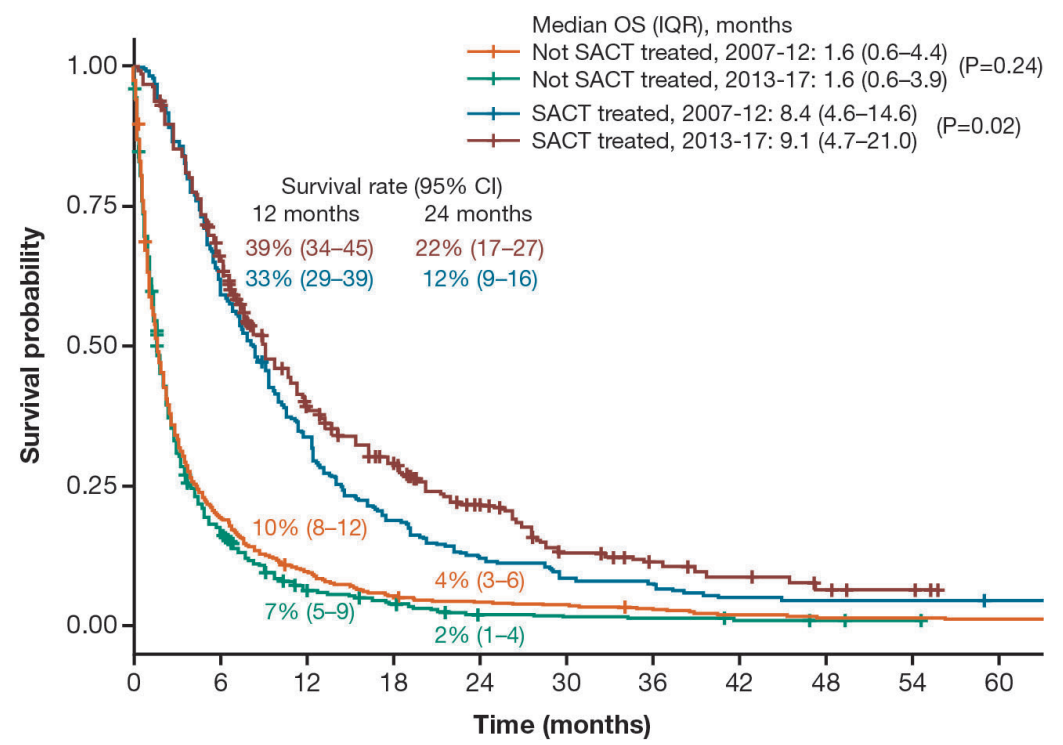

Number at risk

$\begin{array}{llcccccccccc}\text { Not SACT treated, 2007-2012 } & 843 & 161 & 80 & 44 & 34 & 30 & 23 & 15 & 11 & 11 & 10 \\ \text { Not SACT treated, 2013-2017 } & 628 & 107 & 35 & 20 & 8 & 6 & <6 & <6 & <6 & <6 & 0 \\ \text { SACT treated, 2007-2012 } & 338 & 209 & 112 & 63 & 40 & 28 & 25 & 17 & 15 & 15 & 14 \\ \text { SACT treated, 2013-2017 } & 310 & 198 & 104 & 70 & 39 & 20 & 14 & 10 & <6 & <6 & 0\end{array}$

Figure 3 Estimated OS for patients diagnosed during two consecutive time periods with stage IIIB-IV NSCLC and receiving or not receiving SACT following diagnosis. NSCLC, non-small cell lung cancer; OS, overall survival; SACT, systematic anticancer therapy.

$>50 \%$ of patients dying within 3 months of diagnosis. For this group, SACT is the only treatment that is likely to improve survival. ${ }^{10}$ However, less than one-third of the population described was treated, predominantly due to older age and high PS at presentation. The high PS at diagnosis was potentially due to a delay between first symptoms and presentation at hospital. The situation is unlikely to improve unless patients are diagnosed earlier (eg, through screening), therefore potentially presenting with lower PS.

Data informing clinicians of the utility of SACT in patients with higher PS (or advanced age) are sparse because these groups are generally under-represented in clinical trials. Guidelines that caution against SACT for patients with PS $>2$ may explain the low treatment rates in this group reported here. ${ }^{10} 11$ The independent effect of age may relate to increased comorbidities in the elderly (including organ failure) and the reluctance of oncologists and/or elderly patients to risk the wider consequences of receiving cytotoxic treatment. A Canadian study reported that poor PS, rapid decline in functional status, patient's wish, physician recommendation and comorbidities were the main reasons for the absence of any SACT treatment in patients diagnosed with metastatic NSCLC in 2010-2011. ${ }^{12}$ For SACT-treated patients with advanced NSCLC, there was some modest improvement in survival over the past decade (2007-2017), likely resulting from the introduction of targeted therapies such as TKIs. ${ }^{5}$ However, survival rates for untreated patients have not changed and increased rates of treatment between 2007-2012 and 2013-2017 are largely confined to patient groups with low PS.

In patients receiving $2 \mathrm{~L}$ therapy, there was a decrease in 2L TKI use between the 2007-2012 and 2013-2017 periods ( $83.2 \%$ vs $40.4 \%$, respectively), which was mainly driven by erlotinib no longer being indicated for patients who did not have the EGFR mutation as of 2015. In 2013-2017, 2L immunotherapy treatment was received by $18.2 \%$ of patients receiving $2 \mathrm{~L}$ therapy, with most of these treatments commencing after immunotherapy reimbursement began in the UK in 2017. It is too soon, in this cohort, to determine the effect of new immunotherapies on outcomes in the routine clinical setting due to their recent availability and reimbursement, but we hypothesise that increased survival at a population level will require therapies and approaches that broaden the cohort currently considered 'treatable' by clinicians.

This study's strength is the analysis of a long-term realworld dataset that has been clinically curated by checking for inconsistencies where they typically arise in order to ensure consistent quality. The availability of patient-level PS and line of therapy data allows a more detailed understanding of the population treated in a large UK regional hospital. A limitation of the study is that data are reported from a single site, Leeds Teaching Hospital NHS Trust; however, the cohort analysed is large and drawn from a reasonably representative population of UK patients. Additionally, as a teaching hospital and tertiary referral centre that provides all secondary care for its local catchment area of approximately 800000 people, this study 
captured all patients diagnosed with NSCLC in the Leeds area. In this study, we analysed factors associated with likelihood of SACT treatment and discussed potential reasons why they may impact the decision to treat; however, a more detailed clinical case review would be required to confirm the suggested reasons.

In conclusion, developments in SACT between 2007 and 2017 resulted in some improvements in survival outcomes for SACT-treated patients; however, treatment rates remain low and the prognosis for patients with advanced NSCLC remains poor. The reasons patients present as described are multifactorial and may include patient-mediated and health system-mediated delays in diagnosis, exacerbated by the aggressive nature of NSCLC.

This work will serve as a baseline for future research investigating the real-world outcomes observed in advanced NSCLC since the approval of immunotherapy and second-generation and third-generation TKIs.

\section{Author affiliations}

${ }^{1}$ Leeds Teaching Hospitals NHS Trust, Leeds, UK

${ }^{2}$ Real-World Insights, IQVIA, London, UK

${ }^{3}$ Epi-Fit, Bordeaux, Nouvelle-Aquitaine, France

${ }^{4} \mathrm{R} \& D$ Medical Affairs, Bristol Myers Squibb, Madrid, Spain

${ }^{5}$ Worldwide Health Economics \& Outcomes Research, Bristol Myers Squibb, Brainel'Alleud, Belgium

${ }^{6}$ Worldwide Health Economics \& Outcomes Research, Bristol Myers Squibb,

Princeton, New Jersey, USA

${ }^{7}$ Leeds Institute for Data Analytics, University of Leeds, Leeds, UK

\section{Correction notice This article has been corrected since it was first published.}

Acknowledgements Professional writing and editorial assistance was provided by Breanne Landry, PhD, of Parexel International, and was funded by Bristol Myers Squibb.

Contributors LL, CC, MJD, JRP and JCO'D conceived and designed the study, with contributions from MS, MT, SC and WS. GH is the lead for the Leeds Teaching Hospital NHS Trust Real world programme, clinical lead for the Patient Pathway Manager (the Electronic Health Record) and Professor of Cancer Medicine and Digital Health (University of Leeds). SC is the senior information officer working with the REAL-Oncology team at Leeds Teaching Hospital NHS Trust. MS provided expertise to REAL-Oncology regarding the management of lung cancer. MT and MR analysed the data. All authors contributed to the interpretation of the data, the drafting/revision of the manuscript; approved the final manuscript; and agreed to be accountable for all aspects of the work.

Funding This work was supported by Bristol Myers Squibb.

Competing interests REAL-Oncology is a collaboration between Leeds Teaching Hospital NHS Trust and IQVIA. Commercial clients of IQVIA include Bristol Myers Squibb (BMS), which funded the project this work is based on. REAL-Oncology retains all operational, scientific and communications controls. GH is an employee of the University of Leeds and holds an honorary contract with Leeds Teaching Hospital NHS Trust. GH leads the Leeds Teaching Hospitals NHS Trust real-world evidence team collaboration with IQVIA and, as part of this collaboration, IQVIA fund the staff who support this work. GH also reports partial grant funding for a collaboration outside this study from IQVIA. MS was an employee at Leeds Teaching Hospital NHS Trust at the time of the study and he holds an honorary contract with Leeds Teaching Hospital NHS Trust. MS, MT and MR are employees of IQVIA. WS and SC are subcontracted to IQVIA and hold honorary contracts with Leeds Teaching Hospital NHS Trust. MS receives consultancy fees from BMS. CC, MJD, JRP and JCO'D are employees of BMS. CC and JRP report stock ownership in BMS. LL was contracted (paid) as consultant by BMS to support the I-0 Optimise initiative and is an employee of Epi-Fit. There are no further conflicts of interest.
Patient and public involvement statement Patients or the public were not involved in the design, conduct, reporting or dissemination plans of our research.

\section{Patient consent for publication Not required.}

Ethics approval This study was undertaken with approval from the UK Health Research Authority via the National Institute of Health Research Integrated Research Approvals System and performed in accordance with Leeds Teaching Hospitals NHS Trust information governance framework. Due to the study design (noninterventional, retrospective, descriptive study using existing patient records), the need for ethics approval was waived. The study was performed in accordance with the Declaration of Helsinki.

Provenance and peer review Not commissioned; externally peer reviewed. Data availability statement No data are available.

Supplemental material This content has been supplied by the author(s). It has not been vetted by BMJ Publishing Group Limited (BMJ) and may not have been peer-reviewed. Any opinions or recommendations discussed are solely those of the author(s) and are not endorsed by BMJ. BMJ disclaims all liability and responsibility arising from any reliance placed on the content. Where the content includes any translated material, BMJ does not warrant the accuracy and reliability of the translations (including but not limited to local regulations, clinical guidelines, terminology, drug names and drug dosages), and is not responsible for any error and/or omissions arising from translation and adaptation or otherwise.

Open access This is an open access article distributed in accordance with the Creative Commons Attribution Non Commercial (CC BY-NC 4.0) license, which permits others to distribute, remix, adapt, build upon this work non-commercially, and license their derivative works on different terms, provided the original work is properly cited, appropriate credit is given, any changes made indicated, and the use is non-commercial. See: http://creativecommons.org/licenses/by-nc/4.0/.

\section{REFERENCES}

1 Arnold M, Rutherford MJ, Bardot A, et al. Progress in cancer survival, mortality, and incidence in seven high-income countries 1995-2014 (ICBP SURVMARK-2): a population-based study. Lancet Oncol 2019;20:1493-505

2 Walters S, Maringe C, Coleman MP, et al. Lung cancer survival and stage at diagnosis in Australia, Canada, Denmark, Norway, Sweden and the UK: a population-based study, 2004-2007. Thorax 2013;68:551-64.

3 Royal College of Physicians. National lung cancer audit annual report, 2018. Available: https://www.rcplondon.ac.uk/projects/ outputs/nlca-annual-report-2018 [Accessed 18 Jun 2019].

4 Spiro SG, Rudd RM, Souhami RL, et al. Chemotherapy versus supportive care in advanced non-small cell lung cancer: improved survival without detriment to quality of life. Thorax 2004;59:828-36.

5 Minguet J, Smith KH, Bramlage P. Targeted therapies for treatment of non-small cell lung cancer--Recent advances and future perspectives. Int J Cancer 2016;138:2549-61.

6 Brahmer J, Reckamp KL, Baas P, et al. Nivolumab versus docetaxel in advanced squamous-cell non-small-cell lung cancer. N Engl J Med 2015;373:123-35.

7 Rosell R, Carcereny E, Gervais R, et al. Erlotinib versus standard chemotherapy as first-line treatment for European patients with advanced EGFR mutation-positive non-small-cell lung cancer (EURTAC): a multicentre, open-label, randomised phase 3 trial. Lancet Oncol 2012;13:239-46.

8 Garon EB, Rizvi NA, Hui R, et al. Pembrolizumab for the treatment of non-small-cell lung cancer. N Engl J Med 2015;372:2018-28.

9 Ekman S, Griesinger F, Baas P, et al. I-O optimise: a novel multinational real-world research platform in thoracic malignancies. Future Oncol 2019;15:1551-63.

10 Reck M, Popat S, Reinmuth N, et al. Metastatic non-small-cell lung cancer (NSCLC): ESMO clinical practice guidelines for diagnosis, treatment and follow-up. Ann Oncol 2014;25:iii27-39.

11 Azzoli CG, Baker S, Temin S, et al. American Society of clinical oncology clinical practice guideline update on chemotherapy for stage IV non-small-cell lung cancer. J Clin Oncol 2009;27:6251-66.

$12 \mathrm{Ko} \mathrm{JJ}$, Tudor R, Li H, et al. Reasons for lack of referral to medical oncology for systemic therapy in stage IV non-small-cell lung cancer: comparison of 2003-2006 with 2010-2011. Curr Oncol 2017;24:e486-93. 